\title{
Topological Tensor Products of Unbounded Operator Algebras on Fréchet Domains
}

\author{
By \\ Wolf-Dieter HeINRICHS*
}

\begin{abstract}
The aim of this paper is to investigate topological properties of unbounded operator algebras $\mathscr{A} \subset L^{+}(D)$ and its stability under the formation of topological tensor products $\mathscr{A}_{1}$ $\otimes_{\alpha} \mathscr{A}_{2}$. It is used the method to define topological properties by operator.
\end{abstract}

\section{§1. Introduction}

The present paper is concerned with the study of the formation of topological tensor products $\mathscr{A}_{1} \otimes_{\alpha} \mathscr{A}_{2}$ of unbounded operator algebras $\mathscr{A}_{i} \subset$ $L^{+}\left(D_{i}\right)$, where $D_{i} \subset H_{i}$ are Fréchet domains of definition, $L^{+}\left(D_{i}\right)$ are the maximal operator ${ }^{*}$-algebras on $D_{i}$ and $\alpha$ is an arbitrary tensor norm. Recall that tensor products of operators play an important role in the quantum field theory.

It is well-known that an operator algebra $\mathscr{A}$ with $\mathscr{C} \subset A \subset \subset L^{+}(D)$ is a (DF)space, if $D$ is a Fréchet space and $\mathscr{C}$ is the space of very continuous operators, see [11]. But the injective tensor product of (DF)-spaces is not a (DF)-space in general. Therefore we will use the concept of (DF)-spaces by operator (DFO). By this condition we obtain good stability properties by taking topological tensor products. The method to define topological properties by operator was introduced by A. Peris for quasinormable spaces and for (DF)-spaces, see [3] and the method was studied for (DF)-spaces with strong dual density condition by the author, see [8]. In Section 3 we will prove that a lot of operator algebras

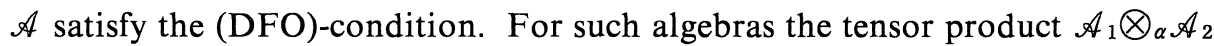
is a (DF)-space.

In Section 4 we will investigate conditions such that $\mathscr{A}_{\text {and }} \mathscr{A}_{1} \bigotimes_{\alpha} \mathscr{A}_{2}$ are bornological (DF)-spaces. For this purpose we need the concept of the density condition (DC) and the strong dual density condition (SDDC). The density

Communicated by Y. Yamasaki, June 19, 1996.

1991 Mathematics Subject Classification(s) : 47D40, 47A67

*Fakultät für Mathematik und Informatik, Universität Leipzig, D-04109 Leipzig, Germany 
condition was introduced by S. Heinrich [5] in the context of ultrapowers of locally convex spaces. The (SDDC) was introduced and investigated by K.D. Bierstedt and J. Bonet in [1] and [2].

It is known that if $L^{+}(D)$ or a dense operator algebra $\mathscr{A}$ is bornological, then $D$ has to satisfy (DC), see [6]. The converse assertion is a tough problem and we need in addition that $D$ has partitions of $I$. Then we get that all ideals $\mathscr{A} \subset L^{+}(D)$ satisfy the strong dual density condition by operator (SDDCO) and have good stability properties by taking tensor products. This implies that $\mathscr{A}$ and $\mathscr{A}_{1} \otimes_{\alpha} \mathscr{A}_{2}$, respectively, are bornological.

\section{§2. Notations and Preliminaries}

The notation for locally convex spaces is standard. If $E$ is a locally convex space, $U(E)$ stands for the system of all absolutely convex closed 0 neighbourhoods in $E$ and $\mathscr{B}(E)$ stands for the system of all absolutely convex bounded sets in $E$. If $V$ is an absolutely convex set, we denote by $p_{V}$ the Minkowski functional of $V$, by $p_{V}^{-1}(0)$ the kernel of $p_{V}$ and if $V$ is in addition absorbent, we denote by $E_{V}$ the quotient $E / p_{V}^{-1}(0)$. Further if $E$ and $F$ are locally convex spaces, then $\mathscr{L}_{b}(E, F)$ denotes the space of all continuous linear mappings from $E$ into $F$ endowed with the topology of uniform convergence on the bounded sets of $E$. We write $\mathscr{L}_{b}(E)$ for $\mathscr{L}_{b}(E, E)$. If $K \subset E, L \subset F$ and $M$ is a linear subspace of $\mathscr{L}_{b}(E, F)$, then we write $W(K, L):=\{T \in M: T(K)$ $\subset L\}$. Let $C_{2}$ denote the space of Johnson. $C_{2}$ is the $l_{2}$-direct sum of the spaces $F_{k}, F_{k}^{\prime}$ with $k \in \mathbf{N}$, where $\left(F_{k}\right)_{k=1}^{\infty}$ is a sequence of finite-dimensional Banach spaces which is dense in the set of all finite-dimensional Banach spaces endowed with the Banach-Mazur distance. If $\alpha$ is a tensor norm, the topology of $E \otimes_{\alpha} F$ is given by the system of seminorms

$$
\left(p_{U} \otimes_{\alpha} q_{V}\right)(z):=\alpha\left(\left(K_{U} \otimes K_{V}\right)(z) ; E_{U}, F_{V}\right) \quad z \in E \otimes F, U \in \mathcal{U}(E), V \in \mathcal{U}(F),
$$

where $K_{U}: E \rightarrow E_{U}$ and $K_{V}: F \rightarrow F_{V}$ are the canonical maps.

Throughout the paper, $D$ denotes a linear subspace of a Hilbert space $H$. We denote the norm and the scalar product of $H$ by $\|\cdot\|$ and $\langle\cdot, \cdot\rangle$, respectively. For a linear operator $T$ on $H$ let $D(T)$ be the domain of definition. The set of linear operators

$$
L^{+}(D):=\left\{T \in \operatorname{End}(D): D \subset D\left(T^{*}\right) \text { and } T^{*}(D) \subset D\right\}
$$

is the maximal Op*-algebra on the domain $D$ with the involution $T^{+}:=\left.T^{*}\right|_{D}$. The domain $D$ will be endowed with the weakest locally convex topology such that $D \ni \varphi \mapsto\left\|T_{\varphi}\right\|$ are continuous seminorms for all $T \in L^{+}(D)$. This topology is called the graph topology $t$. We assume that $D[t]$ is a Fréchet space and in 
this case we say that $D$ is an (F)-domain. Throughout this paper, we fix sequences $\left(A_{k}\right)_{k=1}^{\infty} \subset L^{+}(D)$ and $\left(U_{k}\right)_{k=1}^{\infty} \subset \mathcal{U}(D)$ for each (F)-domain $D$ such that, see $[10]$ :

1. $A_{1}:=I_{D}, A_{k}=A_{k}^{+},\left\|A_{k}^{2} \varphi\right\| \leq\left\|A_{k+1}^{2} \varphi\right\|$.

2. $U_{k}:=\left\{\varphi \in D:\left\|A_{k}^{2} \varphi\right\| \leq 1\right\}$ and $\left(U_{k}\right)_{k=1}^{\infty}$ is a basis of 0 -neighbourhoods in $D$.

Let $D_{b}^{+}$denote the space of all continuous conjugate linear functionals on $D$, endowed with the topology of uniform convergence on bounded subsets of $D$. We fix subsets $N_{k}:=U_{k}^{\circ} \subset D^{+}$for each (F)-domain $D$. Then $\left(N_{k}\right)_{k=1}^{\infty}$ is an increasing fundamental sequence of bounded sets of the (DF)-space $D_{b}^{+}$. We always identify $f \in H$ with a linear functional on $D$. Then we have dense continuous inclusions $D \subset H \subset D_{b}^{+}$.

We define

$$
\mathscr{C}\left(D_{b}^{+}, D\right):=\left\{T \in \mathscr{L}_{b}\left(D, D_{b}^{+}\right): \exists S \in \mathscr{L}\left(D_{b}^{+}, D\right) \forall \varphi \in D T \varphi=S \varphi\right\}
$$

and abbreviate $\mathscr{C}\left(D_{b}^{+}, D\right)$ by $\mathscr{C}$. The elements of $\mathscr{C}$ are called very continuous operators and $\mathscr{C}$ is a *-ideal in $L^{+}(D)$. F stands for the space of all finite rank operators belonging to $L^{+}(D)$. The spaces $\mathscr{F} \subset \mathscr{C} \subset L^{+}(D) \subset \mathscr{L}_{b}\left(D, D_{b}^{+}\right)=: \mathscr{L}$ are endowed with the topology of uniform convergence on bounded sets. The closure of $\mathscr{F}$ in $L^{+}(D)$ is denoted by $\mathscr{V}$.

Let $T$ be an operator in $L^{+}(D) \subset \mathscr{L}$. Then there is an extension $\widetilde{T}$ of the operator $T$ which belongs to $\mathscr{L}\left(D_{b}^{+}, D_{b}^{+}\right)$by $\widetilde{T}:=\left(T^{+}\right)^{\prime}$. Clearly $(\widetilde{T} \varphi)(\psi)=$ $\varphi\left(T^{+} \psi\right)=\left\langle\varphi, T^{+} \psi\right\rangle=\langle T \varphi, \psi\rangle=(T \varphi)(\psi)$ for $\varphi \in D \subset D^{+}$and $\phi \in D$. By [11], Proposition 2.4.c) the sequence $\left(\widetilde{A}_{k}^{2}\left(U_{H}\right)\right)_{k=1}^{\infty}$ is a fundamental sequence of bounded subsets of $D_{b}^{+}$and we have $N_{k}=U_{k}^{\circ}=\widetilde{A}_{k}^{2}\left(U_{H}\right)$.

For more information about unbounded operator algebras see [10], [11] and [13].

\section{§3. The (DF)-Property by Operator for Unbounded Operator Algebras}

In this section we will prove several propositions about permanence of the (DF)-property by taking topological tensor products of unbounded operator algebras on (F)-domains. K.-D. Kürsten proved in [11] that the very continuous operators $\mathscr{C}$ and the ${ }^{*}$-algebra $L^{+}(D)$ are (DF)-spaces. We will obtain that these spaces are (DF)-spaces by operator and have good stability properties by taking tensor products. (DF)-spaces by operator were investigated in $[8]$ and [12].

Definition 3.1. A locally convex space $E$ with an increasing fundamental sequence $\left(M_{k}\right)_{k=1}^{\infty}$ of bounded sets is said to be (DF)-space by operator, 
abbreviated by (DFO)-space, if

$$
\begin{array}{r}
\forall\left(\lambda_{k}\right)_{k=1}^{\infty} \text { pos. } \forall\left(U_{n}\right)_{n=1}^{\infty} \subset \mathcal{U}(E) \exists U \in \mathcal{U}(E) \exists\left(S_{k}\right)_{k=1}^{\infty} \subset \mathscr{L}(E): \\
S_{k}(U) \subset \lambda_{k} M_{k} \text { and }\left(I_{E}-\sum_{k=1}^{n} S_{k}\right)(U) \subset U_{n} \quad k, n \in \mathbb{N} .
\end{array}
$$

Remark. From the characterization of (DF)-spaces given by Bierstedt and Bonet in [2], Lemma 5.A., we deduce that a (DFO)-space is always a (DF)space :

A locally convex space is a (DF)-space if and only if it has an increasing fundamental sequence $\left(M_{k}\right)_{k=1}^{\infty}$ of bounded sets such that for every positive sequence $\left(\varepsilon_{k}\right)_{k=1}^{\infty}$ and for every sequence $\left(U_{n}\right)_{n=1}^{\infty}$ of 0 -neighbourhoods in $E$, there is $U \in \mathcal{U}(E)$ such that

$$
U \subset \bigcap_{n=1}^{\infty}\left(\sum_{k=1}^{n} \varepsilon_{k} M_{k}+U_{n}\right)
$$

Now we give a characterization of the (DFO)-property for reflexive spaces, see [8], Theorem 2.3.

Proposition 3.2. Let $E$ be a reflexive $(D F)$-space. Then the following assertions are equivalent :

1. $E$ is a (DFO)-space.

2. $X \otimes_{\varepsilon} E$ (and $L_{b}(X, E)$, resp.) is a (DF)-space for each Banach space $X$.

It is clear that a Banach space is always a (DFO)-space. For examples and counter-examples see [8]. By Lemma 5 and Proposition 7 in [12] we have the following

Proposition 3.3. If $\alpha$ is a tensor norm and $E, F$ are (DFO)-spaces, then $E \otimes_{\alpha} F$ is a (DF)-space.

The next theorem states that $D_{b}^{+}$is a (DFO)-space and it gives more information about the operators $\left(S_{k}\right)_{k=1}^{\infty}$ if $D$ is an (F)-domain.

Theorem 3.4. Let $D$ be an (F)-domain. Then $D_{b}^{+}$is a (DFO)-space and the corresponding operators are continuous extensions of very continuous operators, i.e. :

$$
\begin{array}{r}
\forall\left(\lambda_{k}\right)_{k=1}^{\infty} \text { pos. } \forall\left(V_{n}\right)_{n=1}^{\infty} \subset \mathcal{U}\left(D_{b}^{+}\right) \exists V \in \mathcal{U}\left(D_{b}^{+}\right) \exists\left(S_{k}\right)_{k=1}^{\infty} \subset \mathcal{C}: \\
\widetilde{S}_{k}(V) \subset \lambda_{k} N_{k} \text { and }\left(I_{D^{+}}-\sum_{k=1}^{n} \widetilde{S}_{k}\right)(V) \subset V_{n} \quad k, n \in \mathbf{N} .
\end{array}
$$


Proof. Let us be given a positive sequence $\left(\lambda_{k}\right)_{k=1}^{\infty}$ and a sequence $\left(V_{n}\right)_{n=1}^{\infty}$ $\subset \mathcal{U}\left(D_{b}^{+}\right)$. Since $D_{b}^{+}$is a (DF)-space, there is some sequence of numbers $\rho_{n}>$ 0 such that $V_{0}:=\bigcap_{n=1}^{\infty} \rho_{n} V_{n}$ belongs to $\mathcal{U}\left(D_{b}^{+}\right)$. Then the polar $V_{0}^{\circ}$ is bounded in $D$ and there exists a positive sequence $\left(\lambda_{k}^{\prime}\right)_{k=1}^{\infty}$ such that

$$
\sum_{k=1}^{\infty} \lambda_{k}^{\prime} \sup \left\{\left\|A_{k} \varphi\right\|^{2}: \varphi \in V_{0}^{\circ}\right\}<1 .
$$

On the linear space

$$
D_{t_{0}}:=\left\{\varphi \in D: \sum_{k=1}^{\infty} \lambda_{k}^{\prime}\left\|A_{k} \varphi\right\|^{2}<\infty\right\}
$$

we define a hermitian sesquilinear form $t_{0}$ by

$$
t_{0}(\varphi, \psi):=\sum_{k=1}^{\infty} \lambda_{k}^{\prime}\left\langle A_{k} \varphi, A_{k} \psi\right\rangle
$$

By K.-D. Kürsten [10], proof of Theorem 4.1, it follows that there exists a positive selfadjoint operator $S_{0}$ acting on the Hilbert space $\bar{D}_{t_{0}} \subset H$ with domain $D_{t_{0}}$ and $\left\langle S_{0} \varphi, S_{0} \psi\right\rangle=t_{0}(\varphi, \psi)$ for $\varphi, \psi \in D_{t_{0}}$. We define $B_{0}:=\left.S_{0}^{-2} \oplus 0\right|_{D}$ $\in \mathscr{C}$ with respect to the orthogonal direct sum $\bar{D}_{t_{0}} \oplus\left(H \ominus \bar{D}_{t_{0}}\right)$ and one sees immediately that $V_{0}^{\circ} \subset \bar{B}_{0}\left(U_{H}\right)$.

Now we choose a sequence $\left(\varepsilon_{k}\right)_{k=1}^{\infty}$ with $0<\varepsilon_{k}<\min \left\{\lambda_{k}, \lambda_{k}^{\prime}\right\}$ and $\varepsilon_{k} N_{k} \subset$ $2^{-k} V_{k}$ for all $k \in \mathbf{N}$. On the linear space

$$
D_{t}:=\left\{\varphi \in D: \sum_{k=1}^{\infty} \varepsilon_{k}\left\|A_{k} \varphi\right\|^{2}<\infty\right\} \supset D_{t_{0}}
$$

we define a hermitian sesquilinear form $t$ once more by

$$
t(\varphi, \psi):=\sum_{k=1}^{\infty} \varepsilon_{k}\left\langle A_{k} \varphi, A_{k} \psi\right\rangle
$$

There exists a positive selfadjoint operator $S$ acting on $\bar{D}_{t} \subset H$ with domain $D_{t}$ and $\langle S \varphi, S \psi\rangle=t(\varphi, \psi)$ for $\varphi, \psi \in D$. We set again $B:=\left.S^{-2} \oplus 0\right|_{D} \in$ $\mathcal{C}$ with respect to the orthogonal direct sum $\bar{D}_{t} \oplus\left(H \ominus \bar{D}_{t}\right)$. Note that $\left.S^{2} \bar{B}\right|_{\bar{D}_{t}}$ $=I_{\bar{D}_{t}}$. For more details relative to this construction see [10]. Since $\bar{D}_{t_{0}} \subset \bar{D}_{t}$, it follows ker $\bar{B} \subset$ ker $\bar{B}_{0}$.

Define $V:=\left\{f \in D_{b}^{+}:\|\widetilde{B} f\| \leq 1\right\} \in \mathcal{U}\left(D_{b}^{+}\right)$and $S_{k}:=\varepsilon_{k} A_{k}^{2} B \in \mathscr{C}$. We conclude

$$
\widetilde{S}_{k}(V) \subset \varepsilon_{k} A_{k}^{2}\left(U_{H} \cap D\right) \subset \varepsilon_{k} N_{k} \subset \lambda_{k} N_{k}
$$

and the first relation of Theorem 3.4 is satisfied. Fix some $n \in \mathbf{N}$. Let $\vartheta \in V$ $\cap \bar{D}_{t}, \psi \in V_{n}^{\circ} \subset D$ and set $\varphi:=\widetilde{B} \vartheta \in U_{H} \cap D\left(S^{2}\right) \subset D$. Since $\varepsilon_{k} A_{k}^{2} \varphi \in \varepsilon_{k} N_{k}$, it follows $\left|\left\langle\varepsilon_{k} A_{k}^{2} \varphi, \psi\right\rangle\right| \leq 2^{-k}$ for all $k \geq n$ by choice of the sequence $\left(\varepsilon_{k}\right)_{k=1}^{\infty}$. By (2) we get 


$$
\begin{aligned}
\left|\left\langle\left(S^{2}-\sum_{k=1}^{n} \varepsilon_{k} A_{k}^{2}\right) \varphi, \psi\right\rangle\right| & =\left|\sum_{k=1}^{\infty} \varepsilon_{k}\left\langle A_{k}^{2} \varphi, \psi\right\rangle-\sum_{k=1}^{n} \varepsilon_{k}\left\langle A_{k}^{2} \varphi, \psi\right\rangle\right| \\
& \leq \sum_{k=n+1}^{\infty} \varepsilon_{k}\left|\left\langle A_{k}^{2} \varphi, \psi\right\rangle\right| \leq \sum_{k=n+1}^{\infty} 2^{-k} \leq 1
\end{aligned}
$$

Further, we have

$$
\begin{aligned}
\left|\left\langle\vartheta-\sum_{k=1}^{n} \widetilde{S}_{k} \vartheta, \psi\right\rangle\right| & =\left|\left\langle S^{2} \widetilde{B} \vartheta-\sum_{k=1}^{n} \varepsilon_{k} A_{k}^{2} \widetilde{B} \vartheta, \psi\right\rangle\right| \\
& =\left|\left\langle\left(S^{2}-\sum_{k=1}^{n} \varepsilon_{k} A_{k}^{2}\right) \varphi, \psi\right\rangle\right| \leq 1
\end{aligned}
$$

and this implies

$$
\left(I-\sum_{k=1}^{n} \widetilde{S}_{k}\right)\left(V \cap \bar{D}_{t}\right) \subset V_{n}^{\circ \circ}=V_{n}
$$

Now let $\vartheta$ be an element in $H \ominus \bar{D}_{t}$. Then $\vartheta \in \operatorname{ker} \bar{B} \subset \operatorname{ker} \bar{B}_{0}$ and this shows $\vartheta \in p_{V_{0}}^{-1}(0)$. By construction of $V_{0}$ we get $\vartheta \in p_{V_{k}}^{-1}(0)$ and $\vartheta \in V_{k}$ for all $k$ $\in \mathbf{N}$. Therefore

$$
\left(I_{D^{+}}-\sum_{k=1}^{n} \widetilde{S}_{k}\right) \vartheta=\vartheta-\sum_{k=1}^{n} \varepsilon_{k} A_{k}^{2} \widetilde{B} \vartheta=\vartheta \in V_{n}
$$

and by (3) a short calculation indicates

$$
\left(I_{D^{+}}-\sum_{k=1}^{n} \widetilde{S}_{k}\right)(V \cap H) \subset V_{n}
$$

Moreover, since $H$ is dense in $D_{b}^{+}, \widetilde{S}_{k}$ are continuous operators and $V_{n}$ is a closed set, we have proved the second relation in the theorem, too.

Theorem 3.5. Let $D$ be an (F)-domain and let $A$ be an algebra of

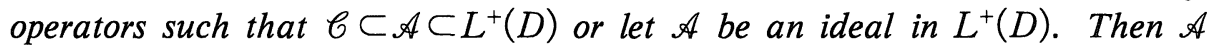
is a (DFO)-space.

Proof. By Theorem 3.4 the space $D_{b}^{+}$is a (DFO)-space, i.e.

$$
\begin{aligned}
\forall\left(\lambda_{k}\right)_{k=1}^{\infty} \text { pos. } & \forall\left(V_{n}\right)_{n=1}^{\infty} \subset \mathcal{U}\left(D_{b}^{+}\right) \exists V \in \mathcal{U}\left(D_{b}^{+}\right) \exists\left(S_{k}\right)_{k=1}^{\infty} \subset \mathcal{C}: \\
& \widetilde{S}_{k}(V) \subset \lambda_{k} N_{k},\left(I_{D^{+}}-\sum_{k=1}^{n} \widetilde{S}_{k}\right)(V) \subset V_{n} \quad k, n \in \mathbf{N} .
\end{aligned}
$$

Let us be given a positive sequence $\left(\lambda_{k}\right)_{k=1}^{\infty}$ and a sequence $\left(M_{k}\right)_{k=1}^{\infty} \subset D$ of bounded subsets. Define $V_{k}:=M_{k}^{\circ} \in \mathcal{U}\left(D_{b}^{+}\right)$and choose $V$ and $\left(S_{k}\right)_{k=1}^{\infty}$ such that (4) is true. Since $U_{k}=N_{k}^{\circ}, M:=V^{\circ} \in \mathscr{B}(D),\left(\widetilde{S}_{k}\right)^{\prime} \in \mathscr{L}(D, D)$ and $f\left(\left(S_{k}\right)^{\prime} \varphi\right)=$ $\left(\widetilde{S}_{k} f\right)(\varphi)=f\left(S_{k}^{+} \varphi\right)$ for $\varphi \in D, f \in D_{b}^{+}$, we see with $R_{k}:=S_{k}^{+}$the following

$$
\begin{array}{r}
\forall\left(\lambda_{k}\right)_{k=1}^{\infty} \text { pos. } \forall\left(M_{n}\right)_{n=1}^{\infty} \subset \mathscr{B}(D) \exists M \in \mathscr{B}(D) \exists\left(R_{k}\right)_{k=1}^{\infty} \subset \mathcal{C}: \\
R_{k}\left(U_{k}\right) \subset \lambda_{k} M,\left(I_{D}-\sum_{k=1}^{n} R_{k}\right)\left(M_{n}\right) \subset M \quad k, n \in \mathbf{N} .
\end{array}
$$


By (4) and (5) the conditions (j) and (jj) are satisfied in the proof of [12], Theorem 13. Therefore, the space $\mathscr{L}=\mathscr{L}_{b}\left(D, D_{b}^{+}\right)$is a (DFO)-space, where the corresponding continuous linear operators $Q_{k}$ on $\mathscr{L}_{b}\left(D, D_{b}^{+}\right)$have the structure

$$
Q_{k}(T):=\sum_{(i, j) \in I_{k}} \widetilde{S}_{i} T R_{j}
$$

where $I_{k} \subset \mathbf{N} \times \mathbf{N}$ is a finite subset and $S_{i}, R_{j} \in \mathscr{C}$. Hence, we get

$$
\begin{gathered}
\forall\left(\varepsilon_{k}\right)_{k=1}^{\infty} \text { pos. } \forall\left(W_{n}\right)_{n=1}^{\infty} \subset \mathcal{U}(\mathscr{L}) \exists W \in \mathcal{U}(\mathscr{L}) \exists\left(Q_{k}\right)_{k=1}^{\infty} \text { with struct. (6): } \\
Q_{k}(W) \subset \varepsilon_{k} \mathcal{M}_{k} \text { and }\left(I_{\mathscr{L}}-\sum_{k=1}^{n} Q_{k}\right)(W) \subset W_{n} \quad k, n \in \mathbf{N} .
\end{gathered}
$$

where $\left(M_{k}\right)_{k=1}^{\infty}$ is an increasing fundamental sequence of bounded sets in $\mathscr{L}$. By construction the linear operators $Q_{k}(A)=\sum \widetilde{S}_{i} A R_{j}$ leave $\mathscr{A}$ invariant. This implies $\left.Q_{k}\right|_{\mathscr{A}} \in \mathscr{L}(\mathscr{A}, \mathscr{A})$ and it is readily seen that $\mathscr{A}$ satisfies an analogous condition to (7) with the increasing fundamental sequence $\left(\mathcal{M}_{k} \cap \mathscr{A}\right)_{k=1}^{\infty}$ of bounded sets. Therefore $\mathscr{A}$ is a (DFO)-space.

Corollary 3.6. Let $D, D_{1}$ and $D_{2}$ be $(F)$-domains, then :

1. The spaces $\mathcal{F}, \mathcal{V}, \mathscr{C}$ and $L^{+}(D)$ are $(D F O)$-spaces.

2. The Calkin algebra $\mathscr{A}_{c}:=L^{+}(D) / \mathscr{V}$ is a (DFO)-space.

3. If $\alpha$ is an arbitrary tensor norm and the algebras $\mathscr{A}_{i} \subset L^{+}\left(D_{i}\right) \quad i=1,2$ satisfy the assumption in Theorem 3.5, then $\mathscr{A}_{1} \otimes_{\alpha} \mathscr{A}_{2}$ and $\mathscr{A}_{1} \widetilde{\otimes}_{\alpha} \mathscr{A}_{2}$ are (DF)-spaces.

Proof. We are going to prove (2). Given a positive sequence $\left(\lambda_{k}\right)_{k=1}^{\infty}$ and a sequence for $\left(V_{k}\right)_{k=1}^{\infty} \subset \mathcal{U}\left(\mathscr{A}_{c}\right)$, there is $\left(W_{k}\right)_{k=1}^{\infty} \subset \mathcal{U}\left(L^{+}(D)\right)$ such that $W_{k}+\mathcal{V}$ $\subset V_{k}$ for $k \in \mathbf{N}$. By Theorem 3.5 we find a $W \subset \mathcal{U}\left(L^{+}(D)\right)$ and $\left(Q_{k}\right)_{k=1}^{\infty} \subset$ $\mathscr{L}\left(L^{+}(D)\right)$ with the structure (6) such that

$$
Q_{k}(W) \subset \varepsilon_{k} M_{k} \text { and }\left(I_{\mathscr{L}}-\sum_{k=1}^{n} Q_{k}\right)(W) \subset W_{n} \quad k, n \in \mathbf{N}
$$

where $\left(M_{k}\right)_{k=1}^{\infty}$ is an increasing fundamental sequence of bounded sets in $\mathscr{L}$. We set $V:=W+\mathscr{V}$ and $\mathcal{N}_{k}:=\mathcal{M}_{k}+\mathcal{V} \in \mathscr{B}\left(\mathscr{A}_{c}\right)$. Since $L^{+}(D)$ is a (DF)-space, the canonical quotient map lifts bounded sets and $\mathcal{N}_{k}$ is a fundamental sequence of bounded sets in $\mathscr{A}_{c}$. We can infer from $Q_{k}(\mathscr{V}) \subset \mathcal{V}$ that the linear operators $\hat{Q}_{k}$ $\in \mathscr{L}\left(\mathscr{A}_{c}, \mathscr{A}_{c}\right)$ are well-defined by $\hat{Q}_{k}(T+\mathscr{V}):=Q_{k}(T)+\mathscr{V}$. Thus

$$
\begin{gathered}
\widehat{Q}_{k}(V)=\hat{Q}_{k}(W+V) \subset Q_{k}(W)+V \subset \varepsilon_{k} \mathcal{M}_{k}+V=\varepsilon_{k} \mathcal{N}_{k}, \\
\left(I_{\mathscr{A}_{c}}-\sum_{k=1}^{n} \widehat{Q}_{k}\right)(V) \subset\left(I_{L^{+}(D)}-\sum_{k=1}^{n} Q_{k}\right)(W)+V \subset W_{n}+V \subset V_{n},
\end{gathered}
$$

and (2) is proven. The assertion (3) follows by Proposition 3.3. 


\section{§4. The Strong Dual Density Condition by Operator for Unbounded Operator Algebras}

The density condition plays an important role in the theory of the maximal Op*-algebras $L^{+}(D)$ on (F)-domains $D$, see [6] and [7]. For example if $D$ is a commutatively dominated (F)-domain, then $L^{+}(D)$ is a bornological space if and only if $D$ satisfies the density condition. In this section we will study the situation for subalgebras $\mathscr{A} \subset L^{+}(D)$ and tensor products of subalgebras. We start with some definitions, see [5] and [1].

Definition 4.1. (1) Let $F$ denote a metrizable space and $\left(U_{k}\right)_{k=1}^{\infty} a$ countable basis of closed absolutely convex 0-neighbourhoods in $F$.

$F$ is said to satisfy the density condition, abbreviated by $(D C)$, if the following holds

$$
\forall\left(\lambda_{k}\right)_{k=1}^{\infty} \text { pos. } \forall n \in \mathbf{N} \exists m \in \mathbf{N} \exists B \in \mathcal{B}(F): \bigcap_{k=1}^{m} \lambda_{k} U_{k} \subset U_{n}+B .
$$

(2) Let $E$ denote a locally convex space with an increasing fundamental sequence $\left(M_{k}\right)_{k=1}^{\infty}$ of bounded sets. $E$ is said to satisfy the strong dual density condition, abbreviated by (SDDC), if the following holds

$$
\forall\left(\lambda_{k}\right)_{k=1}^{\infty} \text { pos. } \forall n \in \mathbf{N} \exists m \in \mathbf{N} \quad \exists U \in \mathcal{U}(E): M_{n} \cap U \subset \Gamma \bigcup_{k=1}^{m} \lambda_{k} M_{k} .
$$

$E$ is said to satisfy the strong dual density condition by operator, $a b$ breviated by (SDDCO), if the following holds

$$
\begin{array}{r}
\forall\left(\lambda_{k}\right)_{k=1}^{\infty} \text { pos. } \forall n \in \mathbb{N} \exists m \in \mathbb{N} \exists U \in \mathcal{U}(E) \exists\left(Q_{k}\right)_{k=1}^{m} \text { lin. op. on } E: \\
\sum_{k=1}^{m} Q_{k}=I_{E} \text { and } Q_{k}\left(M_{n} \cap U\right) \subset \lambda_{k} M_{k}, k=1, \ldots, m .
\end{array}
$$

Quasinormable Fréchet spaces and Fréchet-Montel spaces are examples for spaces satisfying (DC), see [5]. For examples and counter-examples of (F)domains with (DC) see [6] and [7]. By taking polars, it follows that the (F)-space $E$ satisfies (DC) if and only if the strong dual $E_{b}^{\prime}$ satisfies (SDDC). Remark that $U_{k}^{\circ}$ is $\sigma\left(E^{\prime}, E\right)$-compact, such that $\Gamma \bigcup_{k=1}^{m} \lambda_{k}^{-1} U_{k}^{\circ}$ is closed. It is readily seen, that (SDDCO) implies (SDDC). We give a characterization of the (SDDCO) property analogous to the (DFO)-property in Section 3. For details see $[8]$, Theorem 1.6.

Proposition 4.2. Let $E$ be a reflexive ( $D F)$-space. The following assertions are equivalent:

1. E satisfies the strong dual density condition by operator (SDDCO).

2. $X \otimes_{\varepsilon} E$ (and $L_{b}(X, E)$, resp.) satisfies the strong dual density condition 
(SDDC) for each Banach space $X$.

Remark. The direction (1) $\Rightarrow(2)$ is valid for arbitrary (DF)-spaces.

If $E$ is a reflexive (DFO)-space, then (SDDC) implies (SDDCO), see [8], Theorem 2.4. Thus (SDDC) and (SDDCO) are equivalent properties for $D_{b}^{+}$. But we know nothing about the linear operators $Q_{k}$. We are going to study this operators for a large class of (F)-domains. The next definition substitutes the properties (1)-(3) in [13], Proposition 4.1.4.

Definition 4.3. Let $D$ denote an $(F)$-domain. $D$ is said to have partitions of $\mathbb{I}$ if the following holds

$$
\begin{array}{r}
\forall\left(\varepsilon_{k}\right)_{k=1}^{\infty} \text { pos. } \exists\left(\varepsilon_{k}^{\prime}\right)_{k=1}^{\infty} \text { pos. } \forall p \in \mathbf{N} \exists m \in \mathbf{N} \exists\left(Q_{k}\right)_{k=1}^{m} \subset L^{+}(D): \\
\sum_{i=1}^{m} Q_{i}=I_{D} \text { and } Q_{k}\left(\varepsilon_{n} U_{k}\right) \subset \bigcap_{i=1}^{p} \varepsilon_{i}^{\prime} U_{i} \quad k=1, \ldots, m .
\end{array}
$$

Proposition 4.4. Let $D$ be a commutatively dominated $(F)$-domain, i.e.

$$
D:=\bigcap_{k=1}^{\infty} D\left(h_{k}(T)\right)
$$

where $T$ is a self-adjoint operator on a Hilbert space $H$ and $h_{k}$ is a sequence of real measurable functions on the spectrum $\sigma(T)$ of $T$ such that $1=h_{1}(t)$ and $h_{k}(t)^{2} \leq h_{(k+1)}(t)$ a.e. for all $k \in \mathbf{N}$, see [13], 4.3. Then $D$ has partitions of $I$.

Proof. Let us be given a positive sequence $\left(\varepsilon_{k}\right)_{k=1}^{\infty}$ and $p \in \mathbf{N}$. We set $\varepsilon_{i}^{\prime}:=\varepsilon_{i}$ and define bounded measurable functions $q_{k}$ on $\sigma(T)$ by

$$
q_{k}(t):=\frac{\varepsilon_{k}^{-1} h_{k}^{2}(t)}{\sum_{i=1}^{p} \varepsilon_{i}^{-1} h_{i}^{2}(t)} \quad t \in \sigma(T), k=1, \ldots, p
$$

and operators by $Q_{k}:=\left.q_{k}(T)\right|_{D}$. Since the domain of $h_{n}(T)$ is invariant for $q_{k}(T)$, it follows that $Q_{k} \in L^{+}(D)$ for $k=1, \ldots, p$. Fix some $k$ and let $\phi \in \varepsilon_{k} U_{k}$, where $U_{j}:=\left\{\varphi \in D:\left\|h_{j}^{2}(T) \varphi\right\| \leq 1\right\}$ for $j \in N$. We set $\vartheta:=\varepsilon_{k}^{-1} h_{k}^{2}(T) \psi \in U_{H} \cap D$. For an arbitrarry $l=1, \ldots, p$ the estimate

$$
h_{l}^{2}(T) q_{k}(T) \psi=\frac{\varepsilon_{k}^{-1} h_{l}^{2}(T) h_{k}^{2}(T)}{\sum_{i=1}^{p} \varepsilon_{i}^{-1} h_{i}^{2}(T)} \psi=\varepsilon_{l} \frac{\varepsilon_{l}^{-1} h_{l}^{2}(T)}{\sum_{i=1}^{p} \varepsilon_{i}^{-1} h_{i}^{2}(T)} \vartheta \in \varepsilon_{l} U_{H}
$$

shows $Q_{k} \psi \in \varepsilon_{l} U_{l}$. This means $Q_{k}\left(\varepsilon_{k} U_{k}\right) \subset \bigcap_{i=1}^{p} \varepsilon_{i} U_{i}$ for $k=1, \ldots, p$. By construction of the operators we get $\sum_{i=1}^{p} Q_{i}=I_{D}$.

Proposition 4.5. Let $D$ be a quasinormable $(F)$-domain, i.e. 


$$
\forall n \in \mathbf{N} \exists m \in \mathbf{N} \forall \varepsilon>0 \quad \exists M \in \mathscr{B}(D): U_{m} \subset \varepsilon U_{n}+M .
$$

Then $D$ has partitions of $I$.

We note that a quasinormable (F)-space always satisfies (DC). Thus a quasinormable (F)-domain is a domain satisfying (DC) with partitions of $I$.

Proof. Let us be given a positive sequence $\left(\varepsilon_{k}\right)_{k=1}^{\infty}$ and the fundamental system $\left(N_{k}\right)_{k=1}^{\infty}$ of bounded sets in $D_{b}^{+}$. We define bounded sets $B_{k}:=N_{k}+\sum_{i=1}^{k}$ $\varepsilon_{i}^{-1} N_{i}$. Since $D$ is a quasinormable (F)-domain, its strong dual satisfies the strict Mackey condition. Thus, we get

$$
\forall n \in \mathbf{N} \exists m(n) \in \mathbf{N} m(n)>n \exists V_{n} \in \mathcal{U}\left(D_{b}^{+}\right): B_{n} \cap V_{n} \subset \varepsilon_{m(n)}^{-1} N_{m(n)} .
$$

By Theorem $3.4 D_{b}^{+}$is a (DFO)-space and we can choose $V \in \mathcal{U}\left(D_{b}^{+}\right)$and $\left(S_{k}\right)_{k=1}^{\infty} \subset \mathscr{C}$ such that

$$
\widetilde{S}_{k}(V) \subset \varepsilon_{k}^{-1} N_{k} \text { and }\left(I_{D^{+}}-\sum_{k=1}^{n} \widetilde{S}_{k}\right)(V) \subset V_{n} k, n \in \mathbf{N} \text {. }
$$

Let $V^{\circ} \subset \bigcap_{i=1}^{\infty} \delta_{i} U_{i}$ and define $\varepsilon_{i}^{\prime}=1+\left|\delta_{i}\right|$ for all $i \in \mathbf{N}$. We fix some $p \in \mathbf{N}$ and set $m:=m(p)$. By (10) and (11) we get

$$
\begin{aligned}
\left(I_{D^{+}}-\sum_{k=1}^{p} \widetilde{S}_{k}\right)\left(N_{p} \cap V\right) & \subset\left(N_{p}-\sum_{k=1}^{p} \widetilde{S}_{k}(V)\right) \cap V_{p} \\
& \subset B_{p} \cap V_{p} \subset \varepsilon_{m}^{-1} N_{m} .
\end{aligned}
$$

By taking polars we find

$$
\begin{aligned}
S_{k}^{+}\left(\varepsilon_{k} U_{k}\right) & \subset V^{\circ} \subset \bigcap_{i=1}^{p} \varepsilon_{i}^{\prime} U_{i} k \in N \text { and } \\
\left(I_{D}-\sum_{k=1}^{p} S_{k}^{+}\right)\left(\varepsilon_{m} U_{m}\right) & \subset U_{p}+V^{\circ} \subset \bigcap_{i=1}^{p} \varepsilon_{i}^{\prime} U_{i} .
\end{aligned}
$$

Now we define linear operators by $Q_{k}:=S_{k}^{+}$for $k=1, \cdots, p ; Q_{k}:=0$ for $k$ $=p+1, \ldots, m-1$ and $Q_{m}:=I_{D}-\sum_{i=1}^{p} S_{i}^{+}$. Then we have $\sum_{i=1}^{m} Q_{i}=I_{D}$ and $Q_{k}\left(\varepsilon_{k} U_{k}\right) \subset \bigcap_{i=1}^{p} \varepsilon_{i}^{\prime} U_{i}$ for $k=1, \ldots, m$.

Proposition 4.6. Let $D$ be an $(F)$-domain with partitions of $I$ and density condition $(D C)$. Then $D_{b}^{+}(S D D C O)$ and the corresponding operators are continuous extensions of operators in $L^{+}(D)$, more exactly

$$
\begin{array}{r}
\forall\left(\lambda_{k}\right)_{k=1}^{\infty} \text { pos. } \exists V \in \mathcal{U}\left(D_{b}^{+}\right) \forall n \subset \mathbf{N} \exists m \in \mathbf{N} \exists\left(Q_{k}\right)_{k=1}^{m} \subset L^{+}(D): \\
\sum_{k=1}^{m} \widetilde{Q}_{k}=I_{D^{+}} \text {and } \widetilde{Q}_{k}\left(N_{n} \cap V\right) \subset \lambda_{k} N_{k} \quad k=1, \ldots, m .
\end{array}
$$

Remark. Assertion (12) is a little bit stronger than (SDDCO), because the quantors " $\forall n$ " and " $\exists V$ " are swapped. It is known that Assertion (12) with 
arbitrary linear operators and (SDDCO) are equivalent properties for (DFO)spaces, $[8]$, Theorem 2.4 , but the concrete operators can differ.

Proof. Let us be given a positive sequence $\left(\lambda_{k}\right)_{k=1}^{\infty}$. We set $\varepsilon_{k}:=\lambda_{k}^{-1}$. Since $D$ has partitions of $I$, we can find a positive sequence $\left(\epsilon_{k}^{\prime}\right)_{k=1}^{\infty}$ such that for all $p$ there exist $m \in \mathbf{N}$ and operators $\left(Q_{k}\right)_{k=1}^{m} \in L^{+}(D)$ such that

$$
\sum_{i=1}^{m} Q_{i}=I_{D} \text { and } Q_{k}^{+}\left(\varepsilon_{k} U_{k}\right) \subset \bigcap_{i=1}^{p} \varepsilon_{i}^{\prime} U_{i} \quad k=1, \ldots, m
$$

By assumption $D$ satisfies the density condition. Since the bounded set in Definition 4.1.(1) of (DC) can be choosen not depending on $n \in \mathbf{N}$, see [5], 1 . 4, we find a bounded set $M \subset D$ such that for all $n \in \mathbf{N}$ there exists $q \in \mathbf{N}$ with

$$
\bigcap_{i=1}^{q} 2 \varepsilon_{i}^{\prime} U_{i} \subset U_{n}+M .
$$

Choose $m$ and the operators in (13) for $p:=q$. Then we infer

$$
\sum_{i=1}^{m} Q_{i}=I_{D} \text { and } Q_{k}^{+}\left(\varepsilon_{k} U_{k}\right) \subset \frac{1}{2}\left(U_{n}+M\right) \quad k=1, \ldots, m .
$$

By taking polars we get $U_{n}^{\circ} \cap M^{\circ} \subset 2\left(U_{n}+M\right)^{\circ} \subset\left(\left(Q_{k}^{+}\right)^{\prime}\right)^{-1}\left(\varepsilon_{k}^{-1} U_{k}^{\circ}\right)$ and with $V:=M^{\circ} \in \mathcal{U}\left(D_{b}^{+}\right)$and $N_{n}=U_{n}^{\circ}$ we conclude that

$$
\left(Q_{k}^{+}\right)^{\prime}\left(N_{n} \cap V\right) \subset \lambda_{k} N_{k} \quad k=1, \ldots, m .
$$

Since $\widetilde{Q}_{k}=\left(Q_{k}^{+}\right)^{\prime}$ and $\sum_{i=1}^{m} \widetilde{Q}_{i}=I_{D^{+}}$, the statement of the proposition follows.

Theorem 4.7. Let $E$ and $G$ be (DFO)-spaces with increasing fundamental sequences of bounded sets $\left(M_{k}\right)_{k=1}^{\infty}$ and $\left(C_{k}\right)_{k=1}^{\infty}$ respectively. Let $E$ satisfy the property

$$
\begin{array}{r}
\forall\left(\lambda_{k}\right)_{k=1}^{\infty} \text { pos. } \exists U \in \mathcal{U}(E) \forall n \in \mathbf{N} \exists m \in \mathbf{N} \exists\left(S_{k}\right)_{k=1}^{m} \subset \mathscr{L}(E): \\
\sum_{k=1}^{m} S_{k}=I_{E} \text { and } S_{k}\left(M_{n} \cap U\right) \subset \lambda_{k} M_{k} \quad k=1, \ldots, m
\end{array}
$$

and let $G$ satisfy a similar relation

$$
\begin{array}{r}
\forall\left(\lambda_{k}\right)_{k=1}^{\infty} \text { pos. } \underset{\exists}{\exists} U^{\prime} \in \mathcal{U}(G) \forall n^{\prime} \in \mathbf{N} \exists m^{\prime} \in \mathbf{N} \exists\left(R_{k}\right)_{k=1}^{m^{\prime}} \subset \mathscr{L}(G): \\
\sum_{k=1}^{m^{\prime}} R_{k}=I_{G} \text { and } R_{k}\left(C_{n^{\prime}} \cap U^{\prime}\right) \subset \lambda_{k} C_{k} \quad k=1, \ldots, m^{\prime} .
\end{array}
$$

Then $\mathscr{L}_{b}\left(G_{b}^{\prime}, E\right)$ is a $(D F)$-space satisfying the strong dual density condition by operator (SDDCO) and the corresponding continuous linear operators $Q_{k}$ on $\mathscr{L}_{b}\left(G_{b}^{\prime}, E\right)$ have the structure

$$
Q_{k}(T)=\sum_{(i, j) \in I_{k}} S_{i} T R_{j}^{\prime} \quad T \in \mathscr{L}_{b}\left(G_{b}^{\prime}, E\right)
$$


where $I_{k} \subset \mathbb{N} \times \mathbb{N}$ is a finite subset.

Proof. Since $E$ and $G$ are (DFO)-spaces, $E$ and $G_{b}^{\prime}$ satisfy the conditions (j) and (jj) in the proof of [12], Theorem 13. Thus $\mathscr{L}_{b}\left(G_{b}^{\prime}, E\right)$ is a (DF)-space. By taking polars of (17) we get for the space $G_{b}^{\prime}$ the following property

$$
\begin{array}{r}
\forall\left(\lambda_{k}\right)_{k=1}^{\infty} \text { pos. } \exists B \in \mathcal{B}\left(G_{b}^{\prime}\right) \forall n^{\prime} \in \mathbb{N} \exists m^{\prime} \in \mathbb{N} \exists\left(R_{k}\right)_{k=1}^{m^{\prime}} \in \mathscr{L}(G): \\
\sum_{k=1}^{m^{\prime}} R_{k}^{\prime}=I_{G^{\prime}} \text { and } R_{k}^{\prime}\left(\lambda_{k}^{-1} V_{k}\right) \subset V_{n^{\prime}}+B \quad k=1, \ldots, m^{\prime},
\end{array}
$$

where $\left(V_{k}\right)_{k=1}^{\infty}$ is a decreasing basis of 0-neighbourhoods in $G_{b}^{\prime}$. Now given a decreasing positive sequence $\left(\varepsilon_{k}\right)_{k=1}^{\infty}$. By induction we define a new decreasing positive sequence $\left(\lambda_{k}\right)_{k=1}^{\infty}$ such that

$$
\lambda_{j} \sum_{i=1}^{j} \lambda_{i} \leq \frac{1}{4} \varepsilon_{j} \quad j \in \mathbf{N} .
$$

Take $U \in \mathcal{U}(E)$ and $B \in \mathscr{B}\left(G_{b}^{\prime}\right)$ such that (16) and (19) are satisfied for $\left(\lambda_{k}\right)_{k=1}^{\infty}$. We fix some $n \in \mathbb{N}$. Choose $\rho_{1}, \rho_{2} \geq 1$ with $M_{n} \subset \rho_{1} U$ and $B \subset \rho_{2} V_{n}$. Because of (16), there exist $m \in \mathrm{N}$ and linear operators $\left(S_{k}\right)_{k=1}^{m} \subset \mathscr{L}(E)$ such that

$$
\sum_{k=1}^{m} S_{k}=I_{E} \text { and } S_{k}\left(\rho_{2} M_{n} \cap U\right) \subset \lambda_{k} M_{k} \quad k=1, \ldots, m
$$

and by (19) there exist $m^{\prime}$ and linear operators $\left(R_{k}\right)_{k=1}^{m^{\prime}} \subset \mathscr{L}(G)$ such that

$$
\sum_{k=1}^{m^{\prime}} R_{k}^{\prime}=I_{G^{\prime}} \text { and } R_{k}^{\prime}\left(\lambda_{k}^{-1} V_{k}\right) \subset \rho_{1}^{-1} V_{n}+B \quad k=1, \ldots, m^{\prime} .
$$

Without loss of generality, we can assume that $m=m^{\prime}$ and we choose

$$
T \in W\left(V_{n}, M_{n}\right) \cap W(B, U) .
$$

By (22), (23) and the choice of $\rho_{1}, \rho_{2}$ it follows

$$
\begin{aligned}
T R_{l}^{\prime}\left(\lambda_{l}^{-1} V_{l}\right) & \subset T\left(\rho_{1}^{-1} V_{n}\right)+T(B) \\
& \subset \rho_{1}^{-1} M_{n}+U \subset 2 U \quad l=1, \ldots, m
\end{aligned}
$$

and

$$
\begin{aligned}
T R_{l}^{\prime}\left(\lambda_{l}^{-1} V_{l}\right) & \subset T\left(\rho_{1}^{-1} V_{n}\right)+T(B) \\
& \subset T\left(V_{n}\right)+T\left(\rho_{2} V_{n}\right) \subset 2 \rho_{2} M_{n} \quad l=1, \ldots, m .
\end{aligned}
$$

The relations (21), (24) and (25) imply

$$
\begin{aligned}
S_{k} T R_{l}^{\prime}\left(\lambda_{l}^{-1} V_{l}\right) & \subset 2 S_{k}\left(\rho_{2} M_{n} \cap U\right) \\
& \subset 2 \lambda_{k} M_{k} \quad k, l=1, \ldots, m .
\end{aligned}
$$

We define continuous linear operators $Q_{k}$ on $\mathscr{L}\left(G_{b}^{\prime}, E\right)$ by 


$$
Q_{k}(T):=\sum_{i=1}^{k-1} S_{i} T R_{k}^{\prime}+\sum_{i=1}^{k} S_{k} T R_{i}^{\prime}
$$

Consequently

$$
\sum_{k=1}^{m} Q_{k}(T)=\sum_{k=1}^{m} \sum_{i=1}^{k-1} S_{i} T R_{k}^{\prime}+\sum_{k=1}^{m} \sum_{i=1}^{k} S_{k} T R_{i}^{\prime}=T
$$

and because of (26) we infer that

$$
\begin{aligned}
Q_{k}(T)\left(V_{k}\right) & \subset \sum_{i=1}^{k-1} S_{i} T R_{k}^{\prime}\left(V_{k}\right)+\sum_{i=1}^{k} S_{k} T R_{i}^{\prime}\left(V_{i}\right) \\
& \subset \sum_{i=1}^{k-1} 2 \lambda_{k} \lambda_{i} M_{i}+\sum_{i=1}^{k} 2 \lambda_{k} \lambda_{i} M_{k} \\
& \subset 4 \sum_{i=1}^{k} \lambda_{k} \lambda_{i} M_{k} \subset \varepsilon_{k} M_{k} .
\end{aligned}
$$

We proved the following: For each decreasing positive sequence $\left(\varepsilon_{k}\right)_{k=1}^{\infty}$ there exists a 0 -neighbourhood $W(B, U)$ such that for each bounded set $W\left(V_{n}\right.$, $M_{n}$ ), we find an $m \in \mathbf{N}$ and linear operators $Q_{k}$ with the structure (18) such that

$$
\sum_{i=1}^{m} Q_{i}=I_{\mathscr{L}\left(G_{b}^{\prime}, E\right)} \text { and } Q_{k}\left(W\left(V_{n}, M_{n}\right) \cap W(B, U)\right) \subset \varepsilon_{k} W\left(V_{k}, M_{k}\right)
$$

for $k=1, \ldots, m$. This means that $\mathscr{L}\left(G_{b}^{\prime}, E\right)$ satisfies (SDDCO) (even a bit stronger, because the quantors are swapped).

If $D$ is an (F)-domain with (DC) and partitions of $I$ and $E=G:=D_{b}^{+}$, then the spaces $E$ and $G$ satisfy the assumption of Theorem 4.7 and the corresponding linear operators $S_{i}, R_{j}$ are elements of $L^{+}(D)$ by Proposition 4.6. It follows that the linear operators $Q_{k}(T)=\sum S_{i} T R_{j}$ leave ideals of $L^{+}(D)$ invariant. This proves the following theorem.

Theorem 4.8. Let $D$ be an $(F)$-domain with partitions of $I$ and $A$ an ideal of $L^{+}(D)$ containing $\mathscr{C}$. Then are equivalent :

1. $D$ satisfies the density condition $(D C)$.

2. A satisfies the strong dual density condition by operator (SDDCO).

3. A is a bornological (DFO)-space.

Proof. $(1) \Rightarrow(2)$ : This direction is obvious by the above arguments, Proposition 4.6 and Theorem 4.7.

$(2) \Rightarrow(3):$ The ideal $\mathscr{A}$ is a (DFO)- and (DF)-space by Theorem 3.5 and (SDDCO) implies (SDDC). But a (DF)-space satisfying (SDDC) is always bornological by [1], 1.4.(c). 
(3) $\Rightarrow(1):$ Since $\mathscr{C} \subset \mathscr{A} \subset \mathscr{L}$ and $\mathscr{C}$ is dense is $\mathscr{L}$, see [10], Theorem 6.1, it follows that the completion of $\mathscr{A}$ is $\mathscr{L}$ and we conclude that $\mathscr{L}$ is barrelled. The assertion follows by [6], Theorem 4.1. $\diamond$

Corollary 4.9. Let $D$ be an $(F)$-domain satisfying $(D C)$ with partitions of I, then :

1. The spaces $\mathcal{F}, \mathcal{V}, \mathcal{C}$ and $L^{+}(D)$ are bornological (DFO)-spaces.

2. The Calkin-algebra $\mathscr{A}_{c}:=L^{+}(D) / \mathscr{V}$ is a bornological (DFO)-space.

We omit the proof.

Theorem 4.10. Let $D_{i}$ be $(F)$-domains with partitions of $I$ and $\mathscr{A}_{i}$ ideals in $L^{+}\left(D_{i}\right)$ containing $\mathscr{C}_{i}$ for $i=1,2$. Further, let $\alpha$ be an arbitrary tensor norm. Then are equivalent:

1. $D_{1}$ and $D_{2}$ satisfy the density condition $(D C)$.

2. $\mathscr{A}_{1} \otimes_{\alpha} \mathscr{A}_{2}$ is a bornological (DF)-space.

Proof. $(1) \Rightarrow(2)$ : Let $E, G$ be (DF)-spaces and $X:=C_{2}$ the space of Johnson. We define a vector valued trace

$$
\operatorname{tr}:\left(E \otimes_{\varepsilon} X^{\prime}\right) \otimes_{\pi}\left(X \otimes_{\alpha} G\right) \rightarrow E \otimes_{\alpha} G
$$

by $\left(u \otimes x^{\prime}\right) \otimes(x \otimes v) \mapsto\left\langle x, \quad x^{\prime}\right\rangle u \otimes v$. Due to A. Defant tr is a surjective topological homomorphism, see [4], Sections 29 and 35. Put $E:=\mathscr{A}_{2}$ and $G:=$ $X=C_{2}$. By Proptsitions 3.3 and 4.2 and the remark after 4.2. $\mathscr{A}_{2} \otimes_{\varepsilon} X^{\prime}$ is a (DF)-space satisfying (SDDC). By [1], 1.4.(c), $\mathscr{A}_{2} \otimes_{\varepsilon} X^{\prime}$ is a bornological (DF)-space. Of course, $X \otimes_{\alpha} X^{\prime}$ is a bornological (DF)-space, too. Since this property is hereditary under taking projective tensor products, see [9], §41.(7) and (8), and under taking quotients, it follows that $X \otimes_{\alpha} \mathscr{A}_{2}$ is a bornological (DF)-space. By the same argument for

$$
\operatorname{tr}:\left(\mathscr{A}_{1} \otimes_{\varepsilon} X^{\prime}\right) \otimes_{\pi}\left(X \otimes_{\alpha} \mathscr{A}_{2}\right) \rightarrow \mathscr{A}_{1} \otimes_{\alpha} \mathscr{A}_{2}
$$

we prove the assertion.

$(2) \Rightarrow(1)$ : Since $\mathscr{A}_{i}$ is a complemntable subspace of $\mathscr{A}_{1} \otimes_{\alpha} \mathscr{A}_{2}$, the ideal $\mathscr{A}_{i}$ is a bornological (DF)-space. Now, we can conclude as $(3) \Rightarrow(1)$ in the proof of Theorem 4.8. $\diamond$

Remark. (1) A computation as in the proof of Theorem 4.7 indicates that $L^{+}\left(D_{1}\right) \otimes_{\epsilon} L^{+}\left(D_{2}\right)$ satisfies (SDDC) if $D_{1}$ and $D_{2}$ are (F)-domains with (DC) and partitions of $I$. 
(2) With the present method, it is possible to study the space $\mathscr{L}_{b}\left(D_{1}\right.$, $\left.L^{+}\left(D_{2}\right)\right)$, where $D_{1}$ and $D_{2}$ are (F)-domains. For example, let $D_{1}$ be the Schwartz space $\mathbf{S}\left(\mathbf{R}^{4}\right)$ and $D:=D_{2}$ an (F)-domain with partitions of $I$ satisfying (DC), then the space $\mathscr{L}_{b}\left(\mathbf{S}\left(\mathbf{R}^{4}\right), L^{+}(D)\right)$ is a bornological (DF)-space by Proposition 4.6 and Theorem 4.7. We note that the Schwartz space is a commutatively dominated (F)-space satisfying (DC).

\section{References}

[1] Bierstedt, K.D. and Bonet, J., Dual density conditions in (DF)-spaces I, Results in Math., 14 (1988), 242-274.

[2] _ Dual density conditions in (DF)-spaces II, Bull. Soc. Roy. Sci. Liège., 57 (1988), 567 $-589$.

[3] Boyd, C. and Peris, A., A projective description of the Nachbin-ported topology, J. Math. Anal. Appl., 197 (1996), 635-657.

[4] Defant, A. and Floret, K., Tensor Norms and Operator Ideals, North-Holland Math. Studies, North-Holland, Amsterdam, 1993.

[5] Heinrich, S., Ultrapowers of locally convex spaces and applications I., Math. Nachr., 118 (1984), 285-315.

[6] Heinrichs, W.-D., The Density Property in Fréchet-Domains of Unbounded Operator *-algebras, Math. Nachr., 165 (1994), 49-60.

[7] On Unbounded Positive *-Representations on Fréchet-Domains, Publ. RIMS, Kyoto Univ., 30 (1994), 1123-1138.

[8] , The Density Condition and the Strong Dual Density Condition by Operator, Collect. Math., to appear.

[9] Köthe, G., Topological Vector Spaces II, Springer Verlag, New York, Heidelberg, Berlin, 1979.

[10] Kürsten, K.-D., The Completion of the Maximal Op*-Algebras on Fréchet Domains, Publ. RIMS, Kyoto Univ., 22 (1986), 151-175.

[11] _ Duality for Maximal Op*-Algebras on Fréchet Domains, Publ. RIMS, Kyoto Univ., 24 (1988), 585-620.

[12] Peris, A. and Rivera, M.J., Localization of Bounded Sets in Tensor Products, Rev. Univ. Complutense Madrid, to appear.

[13] Schmüdgen, K., Unbounded Operator Algebras and Representations, Akademie-Verlag, Berlin, 1990. 
